\title{
Coherence controlled soliton interactions
}

\author{
Ting-Sen Ku, ${ }^{1}$ Ming-Feng Shih, ${ }^{1}$ Andrey A. Sukhorukov, ${ }^{2}$ and Yuri S. Kivshar ${ }^{2}$ \\ ${ }^{1}$ Department of Physics, National Taiwan University, Taipei, 106, Taiwan \\ ${ }^{2}$ Nonlinear Physics Centre, Research School of Physical Sciences and Engineering, \\ Australian National University, Canberra ACT 0200, Australi ${ }^{*}$
}

\begin{abstract}
We demonstrate theoretically and subsequently observe in experiment a novel type of soliton interaction when a pair of closely spaced spatial optical solitons as a whole is made partially incoherent. We explain how the character of the soliton interaction can be controlled by the total partial incoherence, and show a possibility to change the soliton interaction from attractive to repulsive, or vice versa, near a certain threshold in the coherence parameter.
\end{abstract}

PACS numbers: 42.65.Tg, 42.65.Jx, 42.25.Kb, 42.25.-p

In recent years, the study of solitons has attracted considerable interest in nonlinear optics as well as in other areas of physics such as Bose-Einstein condensates [1]. Usually, most features of solitons are associated with their coherent nature, and the soliton parameters such as the amplitude, phase, and frequency are well defined, being also responsible for the strength and character of the soliton interactions in nonlinear media.

However, the fundamental concept of solitons as fully coherent objects was extended in 1996 to cover more general classes of self-trapped beams which are partially coherent, and therefore can be termed as incoherent solitons [2]. Somewhat related concepts have been developed in the theory of self-trapping of quasi-periodic waves [3] and spontaneously generated temporal solitons in a nonlinear medium with instantaneous response [4].

To support incoherent spatial solitons, the nonlinearity must be noninstantaneous, such that the medium is insensitive to the fast changing phase of the light beam, but only can respond to the time-averaged light intensity. Most experiments on the incoherent spatial solitons have been performed in the biased photorefractive material, whose nonlinear response is noninstantaneous due to the fact that its nonlinearity comes from the migration of space charges [2]. In parallel to the experimental discovery, several different theoretical approaches [5, 6, 7] (for an overview of different methods, see Ref. [1]) to describe incoherent spatial solitons have been developed in the same period of time. One of the theoretical methods, the coherent density approach [5], is a powerful tool for this kind of analysis, and it is used mostly when the dynamics of solitons is the primary subject of study.

Another phenomenon associated with the properties of optical solitons that also caught much research attention is their particle-like interaction [8]. This has been demonstrated, in particular, with the bright optical spatial solitons in self-focusing media. The main features and mechanisms of the interaction of solitons as coherent objects are well known [9]. If two bright solitons are mutually coherent, they attract (repel) each other when they are in-phase ( $\pi$ out of phase). With the relative phase between the interacting solitons being other than zero or $\pi$, there is energy transfer from one soliton to the other, in addition to the repulsive or attractive interaction. This energy transfer is most significant when the soliton relative phase is $\pi / 2$. On the other hand, if the interacting solitons are mutually incoherent or both solitons are partially coherent (the relative phase between them varies much faster than the material can respond), the soliton interaction is always attractive [10].

In this Letter, we reveal the existence of a new type of soliton interaction that is observed when two interacting solitons as a whole are made partially incoherent. We find that the soliton interaction dynamics can become dramatically different from the case when the solitons are mutually coherent. The interaction strength can be controlled by the total coherence. Most remarkably, the interaction may change from attractive to repulsive near a certain threshold in the coherence parameter, or vice versa.

In order to shed light on this novel type of the soliton interaction and its origin, first we present our numerical results, then provide a qualitatively physics intuition to explain why this happens. Finally, we demonstrate experimentally the coherence controlled soliton interaction in a biased self-focusing photorefractive crystal.

In this paper, we use the coherence density approach [5] and perform numerical simulations of interacting partially coherent solitons in a planar geometry. Propagation of light beams in a slow Kerr-type nonlinear medium can be characterized by interaction of many mutually incoherent components governed by a set of coupled nonlinear equations,

$$
i \frac{\partial E_{n}}{\partial z}+D \frac{\partial^{2} E_{n}}{\partial x^{2}}+\gamma \sum_{m}\left|E_{m}\right|^{2} E_{n}=0
$$

where $n$ is the number of component, $\sum_{n}\left|E_{n}\right|^{2}=I$ is the total beam intensity, $x$ and $z$ are the transverse and propagation coordinates normalized to their characteristic values $x_{0}$ and $z_{0}$, respectively, $D=\lambda z_{0} /\left(4 n_{0} x_{0}^{2}\right)$ is the diffraction coefficient, $\lambda$ is the vacuum wavelength, $n_{0}$ is the medium refractive index, and $\gamma$ is the effective normalized nonlinear coefficient. 
When at the crystal input the light is generated by a partially incoherent source, one can associate different components with the same coherent field $E_{s}(x)$, but having different inclination angles $n \theta_{s}$ :

$$
E_{n}(z=0)=\sqrt{G\left(n \theta_{s}\right) \theta_{s}} E_{s}(x) \exp \left(i n \theta_{s} x k_{0}\right),
$$

where $k_{0}=2 \pi x_{0} n_{0} / \lambda$, and $\theta_{s}$ is a discrete step over angles (in radians) which should be chosen sufficiently small. We consider a Gaussian angular power distribution with a width $\theta_{0}$,

$$
G(\theta)=\left(\sqrt{2} / \sqrt{\pi} \theta_{0}\right) \exp \left(-2 \theta^{2} / \theta_{0}^{2}\right)
$$

so that the beams are fully coherent for $\theta_{0} \rightarrow 0$, and become less coherent as $\theta_{0}$ is increased.

We study a mutual interaction between solitons which are excited by two beams launched in parallel,

$$
E_{s}(x)=A\left\{\operatorname{sech}\left[(x+d) / W_{0}\right]+e^{i \phi} \operatorname{sech}\left[(x-d) / W_{0}\right]\right\},
$$

where $2 d$ is the separation between the beams at the input, $W_{0}$ defines the beam widths, $\phi$ is the relative phase between the two beams, and $A=\sqrt{2 D / \gamma W_{0}^{2}}$ is a coherent soliton amplitude.

It is known that two equal-amplitude interacting coherent solitons can experience attraction, repulsion, or energy exchange, and all such processes are controlled by a single parameter, the soliton relative phase $\phi$. As we show below, the interaction of partially coherent beams depends critically on the coherence parameter $\theta_{0}$.

In the simulations, which are performed to supplement the experimental results presented below, we set $\lambda=532 \mathrm{~nm}, n_{0}=2.35, W_{0}=5 \mu \mathrm{m}, 2 d=20 \mu \mathrm{m}$, and the total propagation length of $25 \mathrm{~mm}$. For the in-phase interaction $(\phi=0)$, Fig. 11(a) shows that the attraction between two solitons makes the solitons launched in parallel cross each other periodically when the entire field is coherent $\left(\theta_{0}=0\right)$. We then increase the coherence parameter $\theta_{0}$ from zero, i.e. make the entire field partially incoherent. Since this raises the power threshold for a soliton formation, we compensate for this effect by increasing the strength of the nonlinearity $\gamma$. Our simulation results show that the crossing period gets longer as the field becomes more incoherent. When $\theta_{0}$ reaches the threshold value of 0.0028 , the solitons become propagating in parallel [Fig. 1(d)] and, finally, when $\theta_{0}$ is larger than the threshold, the solitons apparently repel each other [Fig. 1(e)].

For the $\pi$-out-of-phase solitons $(\phi=\pi)$, the situation is just opposite. Figures $1(f-j)$ show that the repulsion between two solitons can be changed to attraction when the incoherence parameter $\theta_{0}$ is larger than 0.0022. Also for the $\pi / 2$-out-of-phase interaction $(\phi=\pi / 2)$, after the interaction, the situation that the right soliton is a bit brighter than the left soliton due to energy transfer when the entire field is coherent $\left(\theta_{0}=0\right)$ can become opposite when $\theta_{0}$ is above a threshold value around 0.0026

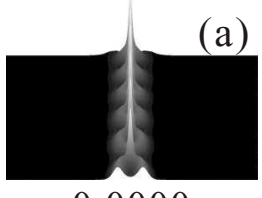

0.0000

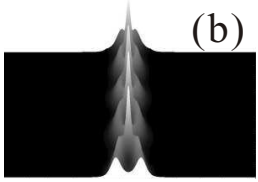

0.0015

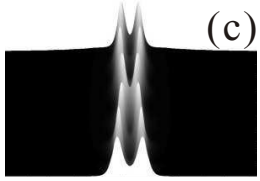

0.0025

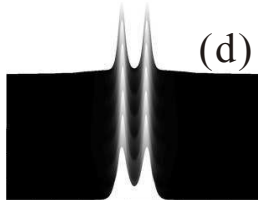

0.0028

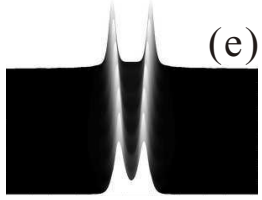

0.0030

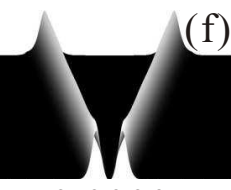

0.0000

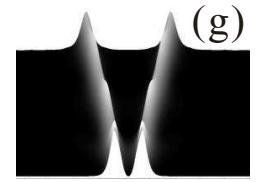

0.0015

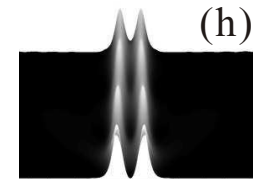

0.0022

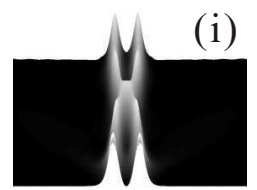

0.0026

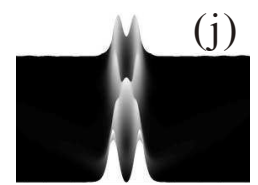

0.0030

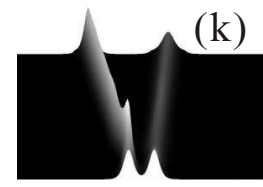

0.0000

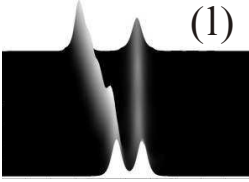

0.0015

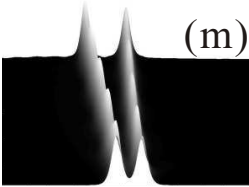

0.0024

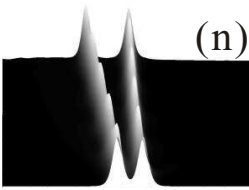

0.0026

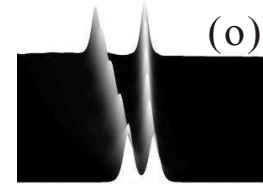

0.0030
FIG. 1: Numerical results. (a)-(e) Interaction of in-phase solitons $(\phi=0)$ for different values of the coherence parameter $\theta_{0}$. (f)-(j) Interaction of $\pi$-out-of-phase solitons $(\phi=\pi)$. (k)-(o) Interaction of $\pi / 2$-out-of-phase solitons $(\phi=\pi / 2)$.

[Fig. 1 $(\mathrm{k}-\mathrm{o})]$, though the opposite energy transfer is not that obvious.

As the next step, we provide a theoretical explanation on how the degree of coherence $\left(\theta_{0}\right)$ can control the soliton interaction. We note that due to the symmetry properties of Eqs. (1), the set of components $E_{n}$ can be chosen in a number of different ways which provide exactly equivalent descriptions [11]. In particular, it is possible to separate out symmetric and anti-symmetric contributions by making a unitary transformation

$$
\widetilde{E}_{n}=\left(E_{-n}+E_{n}\right) / \sqrt{2}=\cos \left(n \theta_{s} x\right) E_{s}(x) \sqrt{2 G\left(n \theta_{s}\right) \theta_{s}},
$$

for $n>0, \widetilde{E}_{0}=E_{0}=E_{s}(x) \sqrt{G(0) \theta_{s}}$, and

$$
\widetilde{E}_{n}=i\left(E_{-n}-E_{n}\right) / \sqrt{2}=\sin \left(n \theta_{s} x\right) E_{s}(x) \sqrt{2 G\left(n \theta_{s}\right) \theta_{s}},
$$

for $n<0$. For a pair of in-phase solitons $(\phi=0)$, the components are symmetric, for $n \geq 0$, and they are antisymmetric, for $n<0$; the situation is reversed for a pair of out-of-phase solitons $(\phi=\pi)$. This symmetry is preserved during the propagation, and therefore there is no 


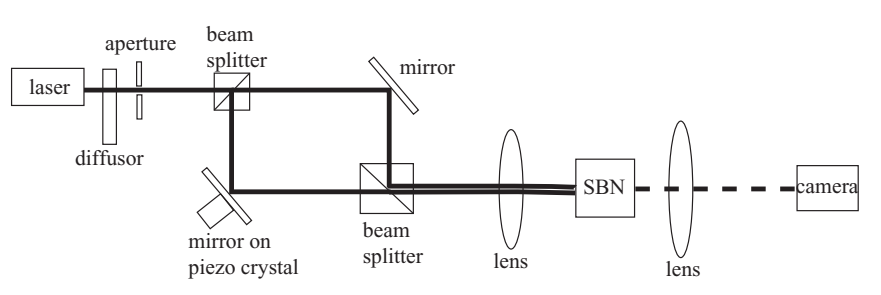

FIG. 2: Sketch of the experimental setup.

net energy exchange between the in-phase or out-of-phase solitons. Thus, the overall effect of attraction or repulsion between the solitons depends on a balance between the powers of symmetric $\left(P_{\mathrm{s}}\right)$ and anti-symmetric $\left(P_{\mathrm{as}}\right)$ components. A specific power ratio $p=P_{\mathrm{s}} / P_{\text {as }}$ is required to observe a stationary propagation of two solitons in parallel, and it can be estimated using an exact two-soliton solution 12] of Eqs. (11) as

$$
p_{0} \simeq 1+4 \exp \left(-2 d / W_{0}\right)
$$

for $2 d \gg W_{0}$. At the crystal input, the parameter $p$ monotonously approaches the value of +1 from above (below) for a pair of in-phase (out-of-phase) solitons as the incoherence parameter $\theta_{0}$ is increased. However, since the partially-coherent input field does not exactly match the soliton profile, some energy is radiated away, and we found that the parameter $p$ calculated for the spatially localized modes may slightly increase or decrease during the initial propagation stage. Because of this radiation mechanism, the condition $p=p_{0}$ can be satisfied at some threshold value of $\theta_{0}^{(\mathrm{th})}$ when the solitons propagate in parallel.

We note that the strongest contribution to the costype soliton components $(n \geq 0)$ comes from the angles around zero, whereas the sin-type components $(n<0)$ are predominantly excited when $n \theta_{s} \simeq \lambda /(4 d)$. Therefore, the soliton separation $2 d$ should be roughly inversely proportional to the threshold value $\theta_{0}^{(\text {th })}$. We have performed additional simulations to verify this prediction and found that when the soliton separation $2 d$ is increased from $20 \mu \mathrm{m}$ to $24 \mu \mathrm{m}$, the threshold value of the coherence parameter is indeed reduced from 0.0028 to 0.0022 , for the interaction of in-phase solitons, and from 0.0022 to 0.0018 , for the $\pi$-out-of-phase solitons.

The interaction of two solitons with the initial $\phi=\pi / 2$ phase shift is more complex. In addition to the effects of attraction or repulsion, the solitons can exchange energy. At the initial propagation stage, the energy flows from the right to the left soliton in components $E_{n}$ with $n \geq 0$, but the flow is opposite for $n<0$ because the phase difference is effectively changed in the corresponding components from $\pi / 2$ to $-\pi / 2$. As the incoherence is increased, the combined powers of two types of components become almost equal, and overall energy exchange is largely suppressed as observed in simulations. (a)
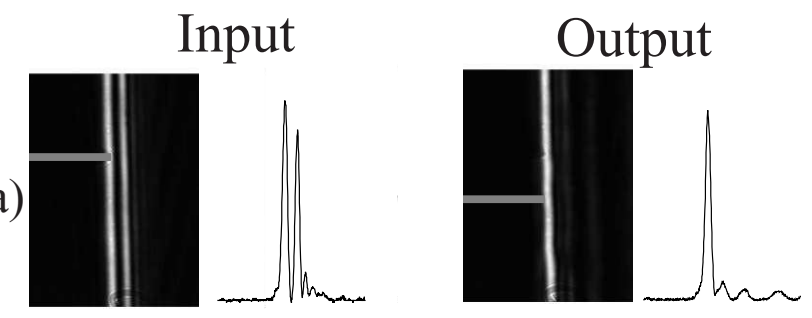

(b)
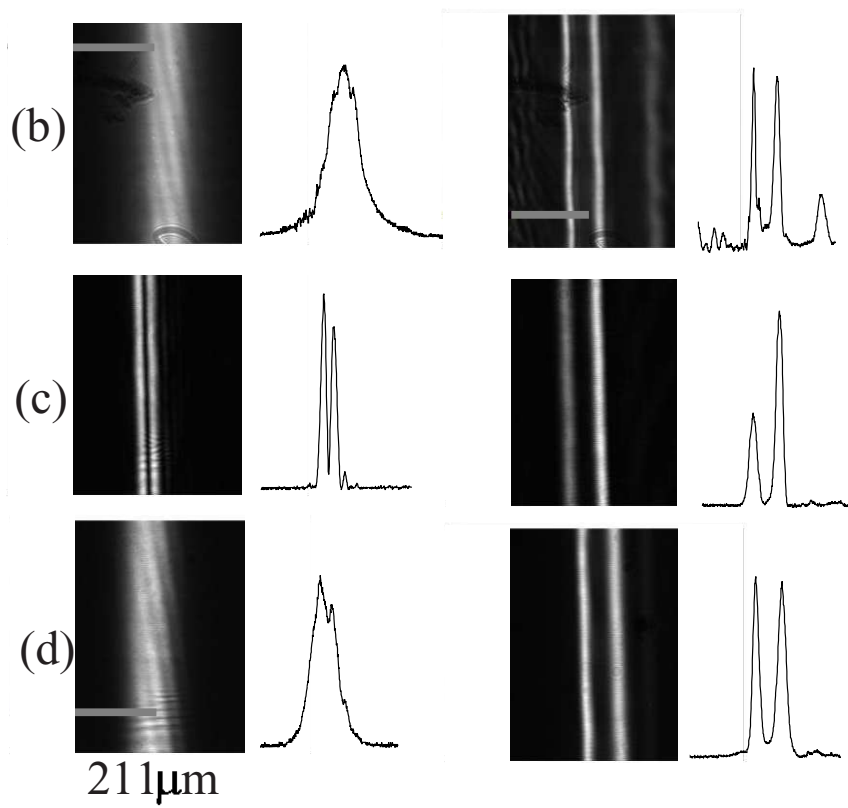

FIG. 3: Experimental results for the interaction of a pair of in-phase solitons for (a) coherent light and (b) incoherent light, and a pair of $\pi / 2$-out-of-phase solitons for (c) coherent light and (d) incoherent light.

In our experiment, we use the interaction of stripe photorefractive solitons 13 as the study object. As schematically shown in Fig. 22 we pass the laser beam through a rotating diffuser to make the light partially incoherent. The light beam is then split into two parts. We then made these two parts to propagate in parallel by using a second beam splitter. These two parts are then focused one-dimensionally by a cylindrical lens onto the front face of a biased photorefractive crystal. The entire crystal is illuminated with a background intensity that is a bit stronger than the soliton intensity to make the nonlinearity close to the Kerr-type nonlinearity 14 but not much stronger to avoid the transverse instability [15].

We first take out the rotating diffuser to make the entire field coherent. We also tune the optical path by applying a suitable voltage on the piezo-transducer attached to the back of one turning mirror to make the two parts in-phase. We then observe, due to the in-phase attraction, these two parts merge into one at the output face of the crystal [Fig. [3(a)]. We keep the optical paths fixed for the entire system, but now insert the rotating diffuser to make the total field partially incoherent. We also increase the nonlinearity correspondingly, by higher 
(a)

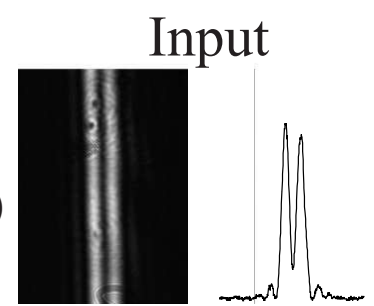

(b)
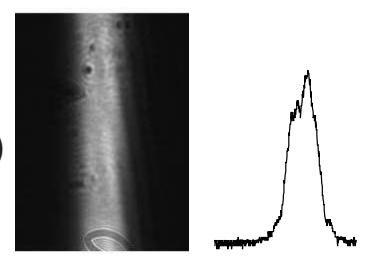

(c)
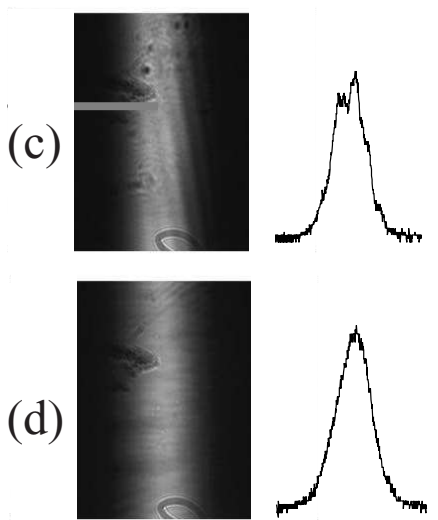

$211 \mu \mathrm{m}$
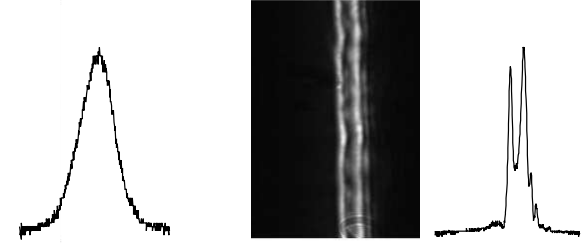

FIG. 4: Experimental results for the interaction of a pair of $\pi$-out-of-phase solitons. The soliton separation at the output face decreases from the most coherent light, in the case (a), to the least coherent light, in the case (d).

biasing voltage, due to the fact that incoherent solitons need higher nonlinearity 2]. As we can see at the output face of the crystal [Fig. 31 b)], these two parts apparently repel each other. This indeed confirms our simulation that if the entire field is made incoherent enough, the attractive interaction can change to a repulsive one. We then tune the relative phase to be $\pi / 2$. When the entire field is coherent, there is strong energy transfer from the left to the right solitons [Fig. 3(c)]. However, when the entire field is partially incoherent enough, we observe the left one is a bit brighter than the right one [Fig. 3(d)], very similar to the simulation results shown in Fig. 1(o).

Finally, we set the relative phase to be $\pi$. We adjust the position of the diffuser and the size of the aperture to make the field to be as coherent as possible. Figure 4(a) shows the repulsion between the two solitons at the output face of the crystal. We then increase the incoherence by opening the aperture at a larger size. As the incoher- ence is increased, the separation become smaller and can be smaller than the separation at the input face, indicating the repulsion have been changed to attraction.

In conclusion, we have described theoretically and demonstrated experimentally a new type of soliton interaction which is observed when two solitons as a whole are made partially incoherent. The interaction strength and type, either attraction or repulsion between the solitons, can be controlled by varying their total coherence. Even more, the interaction may change from attractive to repulsive near a certain threshold in the coherence parameter. We believe this novel type of the coherence controlled soliton interaction is generic for the interaction of partially coherent waves in nonlinear media, and it can be found in other fields.

This work was supported by a collaborative program between the Australian Academy of Science and the National Science Council of Taiwan, and by the Australian Research Council. One of the authors (Yu.K.) thanks the Physics Department of the National Taiwan University for hospitality.

* URL: http://www.rsphysse.anu.edu.au/nonlinear

[1] Yu. S. Kivshar and G. P. Agrawal, Optical Solitons: From Fibers to Photonic Crystals (Academic Press, San Diego, 2003).

[2] M. Mitchell, Z. Chen, M. F. Shih, and M. Segev, Phys. Rev. Lett. 77, 490 (1996).

[3] C. B. Clausen, Yu. S. Kivshar, O. Bang, and P. L. Christiansen, Phys. Rev. Lett. 83, 4740 (1999).

[4] A. Picozzi, M. Haelterman, S. Pitois, and G. Millot, Phys. Rev. Lett. 92, 143906 (2004).

[5] D. N. Christodoulides, T. H. Coskun, M. Mitchell, and M. Segev, Phys. Rev. Lett. 78, 646 (1997).

[6] M. Mitchell, M. Segev, T. H. Coskun, and D. N. Christodoulides, Phys. Rev. Lett. 79, 4990 (1997).

[7] B. Hall, M. Lisak, D. Anderson, R. Fedele, and V. E. Semenov, Phys. Rev. E 65, 035602 (2002).

[8] G. I. Stegeman and M. Segev, Science 286, 1518 (1999).

[9] J. P. Gordon, Opt. Lett. 8, 596 (1983).

[10] D. Anderson and M. Lisak, Phys. Rev. A 32, 2270 (1985).

[11] M. Soljacic, K. Steiglitz, S. M. Sears, M. Segev, M. H. Jakubowski, and R. Squier, Phys. Rev. Lett. 90, 254102 (2003).

[12] T. Kanna and M. Lakshmanan, Phys. Rev. Lett. 86, 5043 (2001).

[13] H. X. Meng, G. Salamo, M. F. Shih, and M. Segev, Opt. Lett. 22, 448 (1997).

[14] M. Segev, G. C. Valley, B. Crosignani, P. Diporto, and A. Yariv, Phys. Rev. Lett. 73, 3211 (1994).

[15] A. V. Mamaev, M. Saffman, D. Z. Anderson, and A. A. Zozulya, Phys. Rev. A 54, 870 (1996). 